\title{
Multiproduct Multiperiod Newsvendor Problem with Dynamic Market Efforts
}

\author{
Jianmai Shi ${ }^{1}$ and Yiping Bao ${ }^{2}$ \\ ${ }^{1}$ College of Information System and Management, National University of Defense Technology, Changsha, Hunan, China \\ ${ }^{2}$ College of Humanities and Social Sciences, National University of Defense Technology, Changsha, Hunan, China \\ Correspondence should be addressed to Jianmai Shi; jianmaishi@gmail.com
}

Received 20 July 2016; Accepted 25 September 2016

Academic Editor: Seenith Sivasundaram

Copyright (c) 2016 J. Shi and Y. Bao. This is an open access article distributed under the Creative Commons Attribution License, which permits unrestricted use, distribution, and reproduction in any medium, provided the original work is properly cited.

\begin{abstract}
We study a multiperiod multiproduct production planning problem where the production capacity and the marketing effort on demand are both considered. The accumulative impact of marketing effort on demand is captured by the Nerlove and Arrow (NA) advertising model. The problem is formulated as a discrete-time, finite-horizon dynamic optimization problem, which can be viewed as an extension to the classic newsvendor problem by integrating with the N-A model. A Lagrangian relaxation based solution approach is developed to solve the problem, in which the subgradient algorithm is used to find an upper bound of the solution and a feasibility heuristic algorithm is proposed to search for a feasible lower bound. Twelve kinds of instances with different problem size involving up to 50 products and 15 planning periods are randomly generated and used to test the Lagrangian heuristic algorithm. Computational results show that the proposed approach can obtain near optimal solutions for all the instances in very short CPU time, which is less than 90 seconds even for the largest instance.
\end{abstract}

\section{Introduction}

In the classic newsvendor problem, the retailer determines the optimal inventory level at the beginning of the sale season when facing an uncertain demand. There is an inventory or handling cost for the unsold products at the end of the season when the prepared inventory is over the actual demand. Usually, there is also a punishing cost for the unmet demand when the inventory level is less than the actual demand during the season. As the enriched practical applications and theoretical meaning, the newsvendor problem has been studied by numerous researchers during last decades. Comprehensive reviews on this topic can be found in works by Khouja [1], Petruzzi and Dada [2], and Qin et al. [3].

In this paper, we investigate a multiperiod production planning problem for multiple products, where the uncertain demand and the accumulated effort of market investment (e.g., advertisement) on demand are considered. The problem is formulated as a discrete-time, finite-horizon dynamic optimization problem, which can be viewed as an extension to the classic newsvendor problem.

Production/inventory management and marketing are both critical to the firm's success. Production-inventory systems are usually controlled with constant safety stock strategy in the situation with sufficient production capacity. However, optimal allocation of production capacity among the multiple products becomes quite important when the capacity is insufficient [4]. Also, production and inventory plans should be optimized according to the dynamic changes of seasonal demands in the planning horizon, which brings great challenges on the model and solution approach. In the other aspect, marketing efforts on the product can create and increase its demand, which simultaneously bring costs along with revenue. In practical business, many firms manage the production-inventory systems as well as the dynamic investment on marketing efforts, such as Huawei in China. Thus, coordinately optimization of production and marketing decisions is critical for this kind of firms and can further 
increase the efficiency for utilizing production capacity and marketing investment, which is a new opportunity for increasing profit.

Most current operations research and management science literatures study production-inventory and marketing problems separately for the newsvendor-type products. A widely used method for formulating the multiperiod production/inventory problem with random demand is to extend the single-period newsvendor problem to a multiperiod one, which is applied to optimize the production-inventory policy over a finite number of periods. Sox and Muckstadt [4] presented an approximate formulation and solution algorithm for the multiproduct multiperiod capacitated production planning problem with random demand, where the market effort is not considered. Matsuyama [5] presented a formulation for the single product multiperiod newsvendor problem, where the initial inventory level of each period is optimized to maximize the overall expected profit. Kogan and Portougal [6] investigate the optimization of control decisions for the multiperiod aggregate production planning problem in a newsvendor framework. Bensoussan et al. [7] investigated a multiperiod newsvendor problem with partially observed demand and presented a dynamic program, through which an optimal feedback ordering policy is obtained. Pan et al. [8] studied the two-period pricing and ordering problem with demand uncertainty in a declining price environment, where the pricing and ordering decisions are controlled by the dominant retailer and can be dynamically optimized based on market demand forecast. Bisi et al. [9] studied the stochastic multiperiod inventory problem where demand in excess of available inventory is lost and unobserved. Thus, the demand data are censored and a Bayesian scheme is used to dynamically update the demand distribution. Xu [10] investigated the optimal myopic inventory policy for the singleproduct multiperiod stochastic inventory problem with batch ordering, where the capacity is purchased as the beginning of the planning horizon. Zhang et al. [11] introduced an online learning method from the field of prediction with expert advice to study the multiperiod newsvendor problem. Kim et al. [12] developed a multistage stochastic programming model for the multiperiod newsvendor problem, which is solved by an extended progressive hedging method. Zhang and Yang [13] investigated a two-product multiperiod stationary newsvendor problem where the two products' total demands are fixed. Cárdenas-Barrón et al. [14] developed a new algorithm based on a reduce and optimize approach and a new valid inequality to solve the multiproduct multiperiod inventory lot sizing with supplier selection problem.

Most of current studies on multiperiod production/ inventory problem with uncertain demand do not consider the impact of dynamic advertisement. In marketing literature, the long-term carryover effects of advertisement are usually described in distributed lag models [15-17]. Berkowitz et al. [18] developed a distributed lag model that estimates the impact of advertising on sales where different media have different lag structures. Herrington and Dempsey [19] compared the current and lagged effects of national-sponsor advertising to that of local and regional sponsors in the automobile industry. Breuer et al. [20] analyzed the short- and long-term effectiveness of different types of online advertising channels by incorporating separate time lags for each advertising channel. Aravindakshan and Naik [21] proposed a general multiple distributed lag framework for estimating Major League Baseball attendance drivers, which focuses specifically on the differential direct and carryover effects of in-game promotions.

Several dynamic advertising models have been developed in marketing literatures, of which the Nerlove-Arrow (NA) model is the most commonly used in theoretical and empirical analyses to estimate carryover effects aggregated by advertisement [21, 22]. N-A model is first proposed by Nerlove and Arrow [23] for describing the demand under the impact of dynamic advertisements, which introduces a definition of the "goodwill" stock to summarize the impacts of current and past advertisement on demand. Then the N-A model is widely suited and extended to construct a variety of demand functions in the marketing and operations management areas [24]. Srinivasan [25] developed a discrete form of the N-A model to optimize advertising media plans for $T$ periods. Bass et al. [26] proposed an extension for N-A model that jointly considers the effects of wearout as well as that of forgetting in the context of multiple advertising themes. Erickson [27] developed an extension for N-A model to formulate the goodwill evolution and presented a price- and advertising-dependent demand function. Aravindakshan et al. [28] extended the Nerlove-Arrow model to incorporate owned and earned media activities along with paid media, which is used to help blood bank marketing managers understand how blood donations can be impacted by managing online media. Most of current literatures on dynamic advertisement concern the optimal policy of dynamic investment on advertisement and their influence on demand, and few of them consider the impact on production and inventory decisions.

Kraiselburd et al. [29] proposed a study that considered the joint optimization of inventory and marketing effort, which is the most relative work we found in the literatures. They investigated a single-period supply chain consisting of a manufacturer and a retailer and developed a methodology for comparing, stocking quantities, marketing effort, and supply chain profits under different scenarios. The demand is assumed to consist of a deterministic component that depends on the marketing effort and an uncertain component that is not a function of channel effort.

As far as we know, there is no literature considering the joint optimization of production and marketing decisions in the dynamic multiperiod scenario. However, production planning and dynamically advertising in a finite horizon with multiple periods and uncertain demand environment become more and more critical for firms to obtain the new profit increasing point in current business world. Motivated by the requirement of practical business and theoretic gap in current literatures, we study the multiproduct multiperiod production planning problem while considering the influence of dynamic advertisement simultaneously.

One of the main contributions of this work is the incorporation of the influence of dynamic advertisement on market demand with the classical newsvendor model and 
developing a new nonlinear programming model for the problem. Also, from a methodology perspective, we integrate the subgradient algorithm and a feasibility search algorithm to solve the arising nonlinear program. Hundreds of randomly generated instances with different sizes are employed to test the computational performance of the proposed solution procedure.

The remainder of the paper is organized as follows: In the next section, we present the nonlinear programming formulation of the joint production and advertising problem. We describe the solution approach in Section 3. In Section 4, we report the computational performance of the solution approach and a two-product numerical example. The paper is concluded in Section 5.

\section{Problem Formulation}

We assume that the demands are uncertain but their distributions are known, though there is not limitation to the type of the distribution. We consider the vendor faces multiple resource constraints, which can change from period to period. As classical newsvendor problem, we also assume the unit overstocking and understocking costs are given, and the stockouts are backordered in the coming periods.

Some notations used in the formulation of our problem are presented in the Notations shown at the end of the paper.

Let $G_{i t}$ denote the customers' goodwill for product $i$ in period $t$ and $Y_{i t}$ the advertising intensity. According to N-A model, goodwill evolves as the following relationship:

$$
G_{i t}=Y_{i t}+\left(1-\delta_{i}\right) G_{i, t-1},
$$

where $\delta_{i}$ is the decay rate of product $i$ representing the decline of memory retention from the previous advertisements.

The excepted demand of product $i$ in period $t$ is assumed to depend on goodwill according to the following function:

$$
D_{i t}=\alpha_{i t}+\beta_{i t} G_{i t} \text {, }
$$

where $\alpha_{i t}$ is an intercept in the linear demand function for product $i$ in period $t$ and $\beta_{i t}$ is a measure of the positive effect of goodwill on demand.

The cumulative demand of product $i$ through period $t$ is uncertain and can be described as

$$
\widetilde{D}_{i t}^{c}=\sum_{j=1}^{t} D_{i j}+u_{i t},
$$

where $u_{i t}$ is a stochastic variable with a known distribution defined on the range $\left[A_{i t}, B_{i t}\right]$.

The holding and backorder cost of product $i$ in period $t$ can be modeled as

$$
\pi_{i t}= \begin{cases}s_{i t}\left(X_{i t}-\widetilde{D}_{i t}^{c}\right), & \widetilde{D}_{i t}^{c} \leq X_{i t} ; \\ g_{i t}\left(\widetilde{D}_{i t}^{c}-X_{i t}\right), & \widetilde{D}_{i t}^{c}>X_{i t} .\end{cases}
$$

In order to facilitate the formulation, we introduce a decision variable

$$
Z_{i t}:=X_{i t}-\sum_{j=1}^{t} D_{i j}
$$

then we can obtain a more convenient formulation for the above model, that is,

$$
\pi_{i t}= \begin{cases}s_{i t}\left(Z_{i t}-u_{i t}\right), & u_{i t} \leq Z_{i t} ; \\ g_{i t}\left(u_{i t}-Z_{i t}\right), & u_{i t}>Z_{i t} .\end{cases}
$$

The expected holding and backorder cost of product $i$ in period $t$ can be calculated as

$$
\begin{aligned}
E\left(\pi_{i t}\right)= & \int_{A_{i t}}^{Z_{i t}} s_{i t}\left(Z_{i t}-u_{i t}\right) f_{i t}\left(u_{i t}\right) d u_{i t} \\
& +\int_{Z_{i t}}^{B_{i t}} g_{i t}\left(u_{i t}-Z_{i t}\right) f_{i t}\left(u_{i t}\right) d u_{i t} .
\end{aligned}
$$

The expected profit of product $i$ in the planning horizon is as follows:

$$
\begin{aligned}
& E_{i}^{\text {profit }}=p_{i}\left\{\int_{A_{i T}}^{Z_{i T}}\left(\sum_{t=1}^{T} D_{i t}+u_{i T}\right) f_{i t}\left(u_{i t}\right) d u_{i t}\right. \\
& \left.+\int_{Z_{i T}}^{B_{i T}}\left(\sum_{t=1}^{T} D_{i t}+Z_{i T}\right) f_{i t}\left(u_{i t}\right) d u_{i t}\right\}-\sum_{t=1}^{T} E\left(\pi_{i t}\right) \\
& \quad-c_{i}\left(\sum_{t=1}^{T} D_{i t}+Z_{i T}\right)-\sum_{t=1}^{T} w_{i} Y_{i t}^{2} .
\end{aligned}
$$

The multiproduct multiperiod production planning problem can be formulated as

$$
\begin{array}{ll}
\max & \Pi=\sum_{i=1}^{I} E_{i}^{\text {profit }} \\
\text { s.t. } & \sum_{i=1}^{I} h_{i}\left(D_{i t}+Z_{i t}-Z_{i, t-1}\right) \leq H_{t}, \quad \forall t, \\
& D_{i t}+Z_{i t}-Z_{i, t-1} \geq 0, \quad \forall i, t, \\
& G_{i t}=Y_{i t}+\left(1-\delta_{i}\right) G_{i, t-1}, \\
& A_{i t} \leq Z_{i t} \leq B_{i t}, \\
& G_{i t} \geq 0 \\
& Y_{i t} \geq 0 .
\end{array}
$$

Objective (8) is to maximize the overall profit for all products in the planning horizon. Constraints (10) ensure that the total capacity consumed in period $t$ should be no more than the available capacity in that period, which come from $\sum_{i=1}^{I} h_{i}\left(X_{i t}-X_{i, t-1}\right) \leq H_{t}$ by substituting (5). Constraints (11) ensure that the cumulative production quantity for product $i$ is nondecreasing, which comes from $X_{i t} \geq X_{i, t-1}$ by substituting (5). Constraints (12) state the dynamic evolution of goodwill for product $i$. Constraints (13)-(15) restrict the ranges for variables. 


\section{Solution Approach}

In this section, we present a solution approach for model (9)-(15) based on the Lagrangian relaxation, in which the subgradient algorithm is applied to solve the Lagrangian dual problem and a feasibility heuristic is used to find a feasible lower bound.

3.1. Lagrangian Relaxation and Decomposition. We make Lagrangian relaxation for constraints (10) and (11); then we can obtain the following function:

$$
\begin{aligned}
L \Pi= & \sum_{i=1}^{I} E_{i}^{\text {profit }} \\
& +\sum_{t=1}^{T} \lambda_{t}\left(H_{t}-\sum_{i=1}^{I} h_{i}\left(D_{i t}+Z_{i t}-Z_{i, t-1}\right)\right) \\
& +\sum_{t=1}^{T} \sum_{i=1}^{I} \eta_{i t}\left(D_{i t}+Z_{i t}-Z_{i, t-1}\right),
\end{aligned}
$$

where $\lambda_{t}$ and $\eta_{i t}$ are the corresponding Lagrangian multipliers for constraints (10) and (11), respectively.

We can see that the Lagrangian relaxation problem can be decomposed into two independent subproblems: a newsvendor-type subproblem and a dynamic advertising subproblem.

\section{The Newsvendor-Type Subproblem}

$$
\begin{aligned}
& \mathrm{NTP}_{i}=\int_{A_{i T}}^{Z_{i T}}\left[p_{i} u_{i T}-s_{i T}\left(Z_{i T}-u_{i T}\right)\right] f_{i T}\left(u_{i T}\right) d u_{i T} \\
& +\int_{Z_{i T}}^{B_{i T}}\left[p_{i} Z_{i T}-g_{i T}\left(u_{i T}-Z_{i T}\right)\right] f_{i T}\left(u_{i T}\right) d u_{i T} \\
& \quad-\left(c_{i}+\lambda_{T} h_{i}-\eta_{i T}\right) Z_{i T} \\
& \quad-\sum_{t=1}^{T-1}\left\{s_{i t} \int_{A_{i t}}^{Z_{i t}}\left(Z_{i t}-u_{i t}\right) f_{i t}\left(u_{i t}\right) d u_{i t}\right. \\
& +g_{i t} \int_{Z_{i t}}^{B_{i t}}\left(u_{i t}-Z_{i t}\right) f_{i t}\left(u_{i t}\right) d u_{i t} \\
& \left.+Z_{i t}\left(\left(-\lambda_{t+1}+\lambda_{t}\right) h_{i}+\eta_{i, t+1}-\eta_{i t}\right)\right\}
\end{aligned}
$$

s.t. (13).

The newsvendor-type subproblem is a combination of $T$ classical newsvendor problems, which can be solved by the following lemma.

Lemma 1. Given the Lagrangian multipliers $\lambda_{t}$ and $\eta_{i t}$, the optimal solutions for the newsvendor-type subproblem (17) are as follows:

$$
\begin{aligned}
& \text { for } t=1,2, \ldots, T-1, \\
& \qquad Z_{i t}^{*}=\frac{g_{i t}+\left(\lambda_{t+1}-\lambda_{t}\right) h_{i}-\eta_{i, t+1}+\eta_{i t}}{g_{i t}+s_{i t}}
\end{aligned}
$$

$$
\begin{aligned}
& \text { and for } t=T \text {, } \\
& \qquad Z_{i T}^{*}=\frac{p_{i}+g_{i T}-c_{i}-\lambda_{T} h_{i}+\eta_{i T}}{p_{i}+g_{i T}+s_{i t}} .
\end{aligned}
$$

The proof of Lemma 1 is presented in the Appendix.

The Dynamic Advertising Subproblem

$$
\begin{aligned}
& \operatorname{DAP}_{i} \\
& \quad=\sum_{t=1}^{T}\left\{\left(p_{i}-c_{i}-\lambda_{t} h_{i}+\eta_{i t}\right)\left(\alpha_{i t}+\beta_{i t} G_{i t}\right)-w_{i} Y_{i t}^{2}\right\}
\end{aligned}
$$

s.t. (12), (14), and (15).

The dynamic advertising subproblem is a discrete $\mathrm{N}$ A model, which can be solved recursively using standard dynamic programming theory. Then we can obtain the proposition for solving this subproblem.

Proposition 2. The optimal solution for the dynamic advertising subproblem is, for $t=1,2, \ldots, T$,

$$
Y_{i t}^{*}=\frac{1}{2 w_{i}} \sum_{j=0}^{T-t} b_{i, t+j}\left(1-\delta_{i}\right)^{j},
$$

and the associated goodwill is

$$
G_{i t}^{*}=\sum_{j=0}^{t-1}\left(1-\delta_{i}\right)^{j} Y_{i, t-j}^{*}+\left(1-\delta_{i}\right)^{t} G_{0}
$$

where $b_{i t}=\beta_{i t}\left(p_{i}-c_{i}-\lambda_{t} h_{i}+\eta_{i t}\right)$.

The proof of Proposition 2 is presented in the Appendix.

Proposition 2 shows that the optimal investment on advertisement in period $t$ is a function of the Lagrangian multipliers starting from period $t$.

3.2. Subgradient Algorithm. Based on the decomposition and the solutions for two subproblems, we employ subgradient algorithm to solve the Lagrangian dual problem. The main procedure of the subgradient algorithm is presented in Algorithm 1.

In each iteration of the subgradient algorithm, given the value of the Lagrangian multipliers, a solution for the relaxed problem can be obtained by Lemma 1 and Proposition 2 . However, this solution may not satisfy constraints (10) and (11), that is, infeasible to the original problem. Thus, a feasibility heuristic, noted as Algorithm 2, is developed to find a feasible solution based on the relaxed solution. The goal of the subgradient algorithm is to find a relaxed upper bound and a feasible lower bound for the original problem. If the gap between the upper bound and the lower bound is small enough, it can be viewed that a near optimal solution is obtained for the original problem.

3.3. Feasibility Heuristic. The feasibility heuristic is used to check if all constraints (10) and (11) are satisfied. If there are 


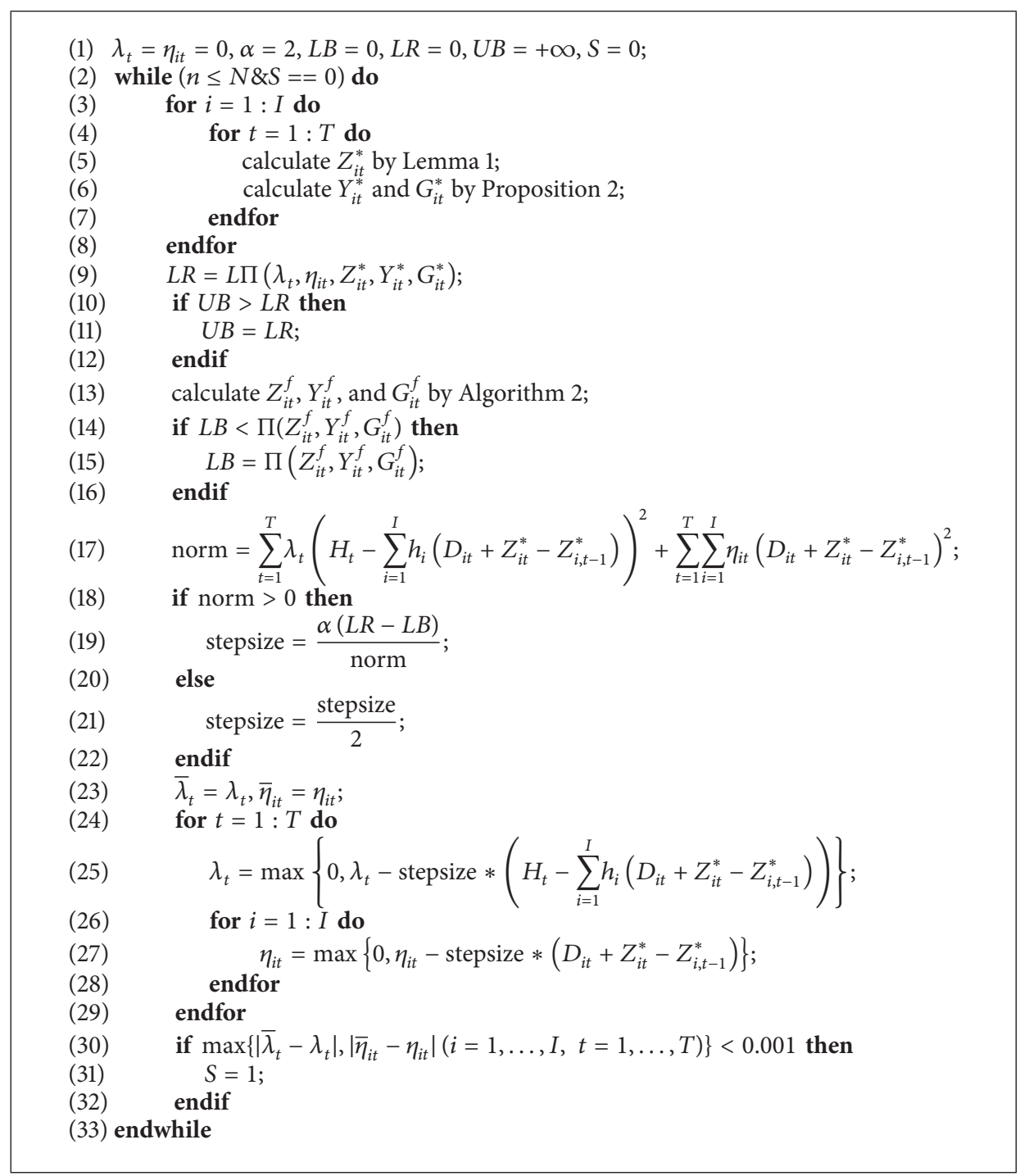

Algorithm 1: Subgradient algorithm.

some constraints that are violated, the heuristic finds them and adjusts the corresponding solution to form a feasible solution for the original problem. The main procedure of the feasibility heuristic is presented in Algorithm 2.

The basic idea of the feasibility heuristic algorithm is to find the unsatisfied constraints one by one and fix them through adjusting the relaxed solution. We first check all the capacity constraints (10) and find out the unsatisfied ones. The production quantities of the products are reduced from the one consuming higher capacity to the lower one until the capacity constraints are satisfied. Then all constraints (11) are checked and the unsatisfied ones are found out. We reduce the production quantity of the former period to make the constraint feasible.

\section{Computational Performance and Managerial Insight}

In order to analyze the performance of the solution approach, we randomly generate 1,200 instances of problem, all of which are solved by the proposed solution approach. The set of tested instances includes four different product lists: 5, 10,25 , and 50 products, respectively. For each product list, different planning horizons including 5,10 , and 15 periods are considered. Thus, there are 12 kinds of instances with different problem size, and 100 instances are randomly generated for each problem size. The demands for all the products are assumed to follow normal distribution. The summarized computational performance for all the instances is presented in Table 1. The computational experiment is conducted on a Lenovo laptop with Intel ${ }^{\circledR}$ Core $^{\mathrm{TM}}$ i5-4200U CPU @ $1.60 \mathrm{GHz}$ $2.30 \mathrm{GHz}$ processor and $8 \mathrm{~GB}$ of RAM. The program is coded in MATLAB.

From Table 1, we can see that our solution approach can present near optimal solutions for most of the instances with different sizes, and the gap between the relaxed upper bound and the feasible lower bound is controlled in $0.1 \%$ expected for some large scale instances with 50 products and 15 periods. Then we checked the computational results for all 


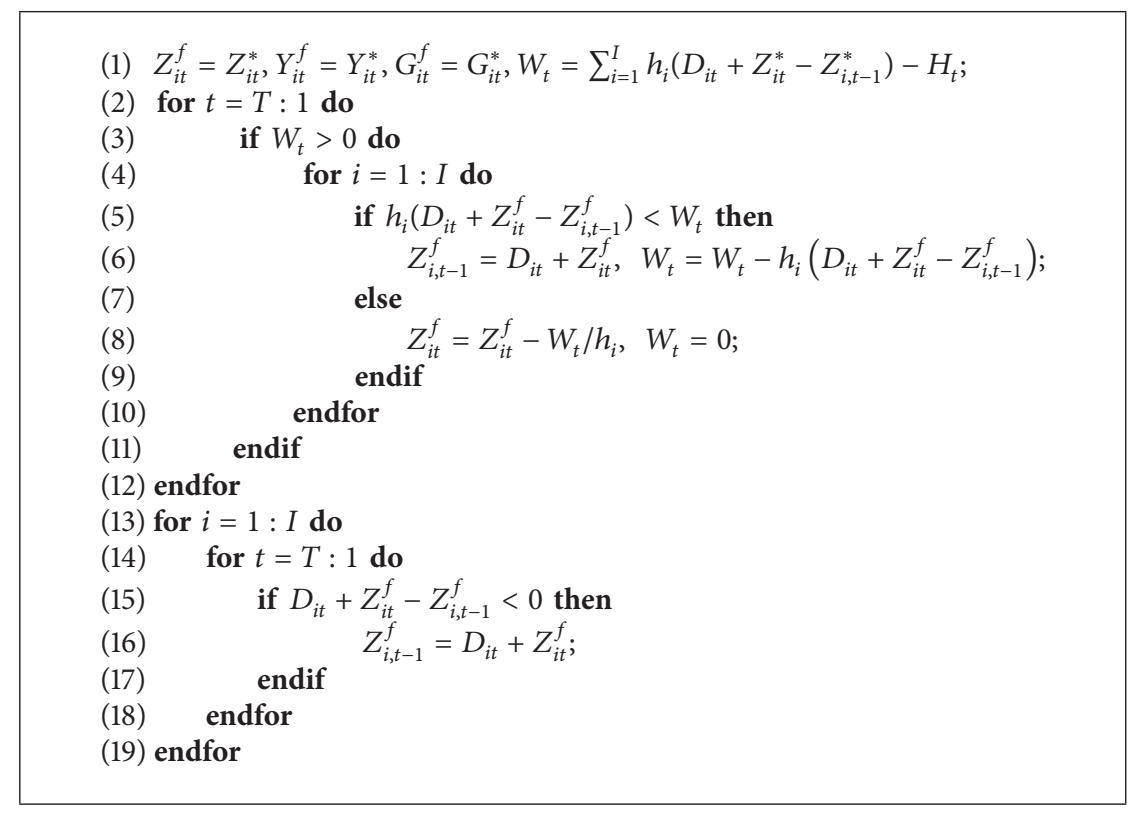

Algorithm 2: Feasibility heuristic.

TABLE 1: The computational performance of the solution approach.

\begin{tabular}{|c|c|c|c|c|c|c|c|}
\hline \multicolumn{2}{|c|}{ Problem size } & \multicolumn{3}{|c|}{$\operatorname{Gap}(E-04)$} & \multicolumn{3}{|c|}{ CPU time (seconds) } \\
\hline$I$ & $T$ & Min & Max & Average & Min & $\operatorname{Max}$ & Average \\
\hline \multirow{3}{*}{5} & 5 & 0.74 & 4.68 & 1.97 & 3.451 & 4.623 & 3.801 \\
\hline & 10 & 1.64 & 5.58 & 2.90 & 6.547 & 7.623 & 6.978 \\
\hline & 15 & 1.20 & 6.34 & 3.10 & 8.835 & 10.17 & 9.328 \\
\hline \multirow{3}{*}{10} & 5 & 0.54 & 2.05 & 1.04 & 4.820 & 7.472 & 5.458 \\
\hline & 10 & 0.80 & 3.41 & 1.56 & 8.440 & 11.185 & 9.301 \\
\hline & 15 & 0.95 & 4.43 & 2.13 & 15.801 & 24.315 & 17.021 \\
\hline \multirow{3}{*}{25} & 5 & 0.31 & 1.01 & 0.67 & 10.826 & 14.086 & 12.167 \\
\hline & 10 & 0.46 & 2.33 & 1.34 & 21.762 & 23.010 & 22.408 \\
\hline & 15 & 0.62 & 4.13 & 1.97 & 33.017 & 53.115 & 37.775 \\
\hline \multirow{3}{*}{50} & 5 & 0.33 & 0.66 & 0.47 & 13.875 & 30.773 & 25.099 \\
\hline & 10 & 0.55 & 1.87 & 1.33 & 44.053 & 62.554 & 48.422 \\
\hline & 15 & 0.53 & 117.98 & 9.62 & 65.901 & 87.031 & 71.839 \\
\hline
\end{tabular}

the one hundred large scale instances for 50 products and 15 periods and found that only 4 instances' gaps are over $0.1 \%$ and reach about $1 \%$, while the other 96 instances' gaps are all below $0.1 \%$. Also, the computational time of the proposed approach is short, and it is no more than 90 seconds even for the large scale instances with 50 products and 15 periods. Thus, the overall performance of the solution approach is good enough and can be used to solve most instances in practical business.

In order to further illustrate the multiproduct multiperiod production planning problem with market effort, we present a two-product ten-period numerical example. The main parameters are presented in Table 2, and the demands for both products in all periods are assumed to follow normal distribution. And the initial goodwill for both products is set to zero.
TABLE 2: Main parameters for the numerical example.

\begin{tabular}{lcccccccc}
\hline \multirow{2}{*}{ Product } & \multicolumn{8}{c}{ Parameters } \\
& $p_{i}$ & $c_{i}$ & $g_{i t}$ & $s_{i t}$ & $a_{i}$ & $\alpha_{i t}$ & $\beta_{i t}$ & $\delta_{i}$ \\
\hline 1 & 120 & 48 & 48 & 6 & 1.5 & 260 & 50 & 0.2 \\
2 & 180 & 72 & 72 & 9 & 2.5 & 180 & 40 & 0.3 \\
\hline
\end{tabular}

There are usually two typical capacity strategies in production planning: dynamic capacity and fixed capacity. In the dynamic capacity strategy, the production capacity is prepared according to the market demand forecast in the planning horizon and the capacity is different in different periods. In the fixed strategy, the production capacity is the same for all periods and the demand is satisfied through adjusting inventory strategy. In the numerical example, we 
TABLE 3: Capacities in each period under different capacity allocation strategies.

\begin{tabular}{|c|c|c|c|c|c|c|c|c|c|c|}
\hline \multirow{2}{*}{ Strategy } & \multicolumn{10}{|c|}{ Period } \\
\hline & 1 & 2 & 3 & 4 & 5 & 6 & 7 & 8 & 9 & 10 \\
\hline Dynamic capacity & 2733 & 3847 & 4633 & 5164 & 5491 & 5642 & 5632 & 5455 & 5098 & 2487 \\
\hline Fixed capacity & 4618 & 4618 & 4618 & 4618 & 4618 & 4618 & 4618 & 4618 & 4618 & 4618 \\
\hline
\end{tabular}

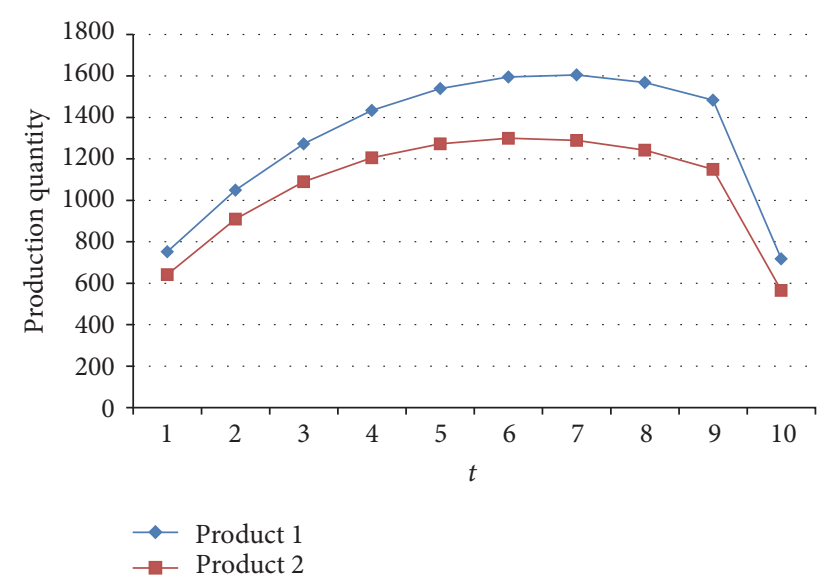

FIgURE 1: The production decisions under dynamic capacity strategy.

let the overall capacity for the planning horizon be 46,180 , while the capacity in each period is allocated according to dynamic and fixed strategies, respectively, which is illustrated in Table 3.

The example is solved under both capacity strategies. Figure 1 presents the production decisions for the two products in all periods under dynamic capacity strategy, while Figure 2 presents the production decisions under the fixed capacity strategy. It can be seen that the production strategy follows an increasing-decreasing pattern when the capacity strategy is dynamic and follows an increasing-steady-decreasing pattern when the capacity strategy is fixed.

Figure 3 presents the advertising decisions for the two products in all periods under dynamic capacity strategy, while Figure 4 presents the production decisions under the fixed capacity strategy. We can see that the advertising intensity follows a decreasing pattern for both dynamic and fixed capacity strategies. However, the advertising intensity decreases more quickly under the fixed capacity strategy.

The overall profit under the dynamic capacity strategy is $1,121,774.78$, while the profit under fixed capacity strategy is $1,104,022.82$. Thus, when the total production capacity is constant, the overall profit can be increased by about $1.61 \%$ if the capacity is dynamically allocated among periods. For firms with huge markets, even one percentage increasing on profit would mean millions of dollars.

We further examine the reasons for causing these differences between dynamic and fixed capacity strategies. The actual consumption of production capacity under both strategies is presented in Figure 5. In the first three periods, the goodwill for the products accumulates due to the investment

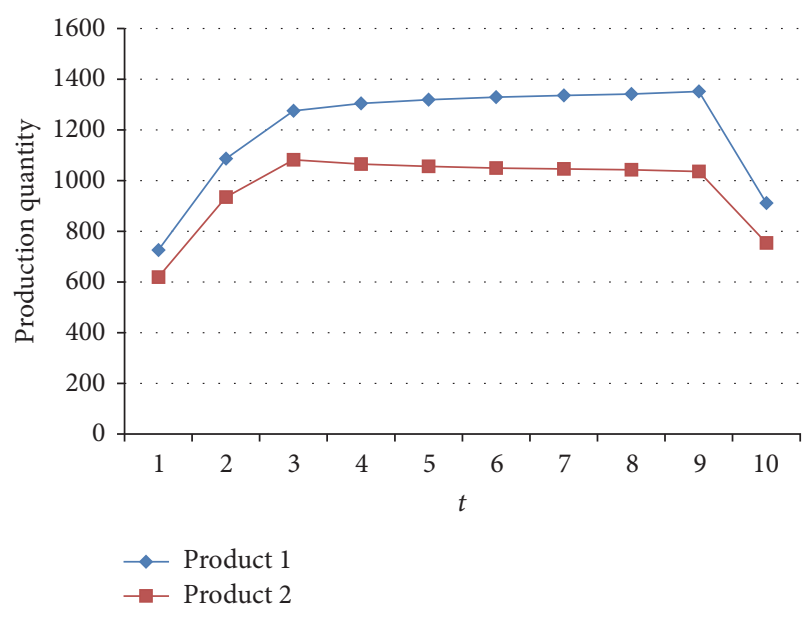

FIGURE 2: The production decisions under fixed capacity strategy.

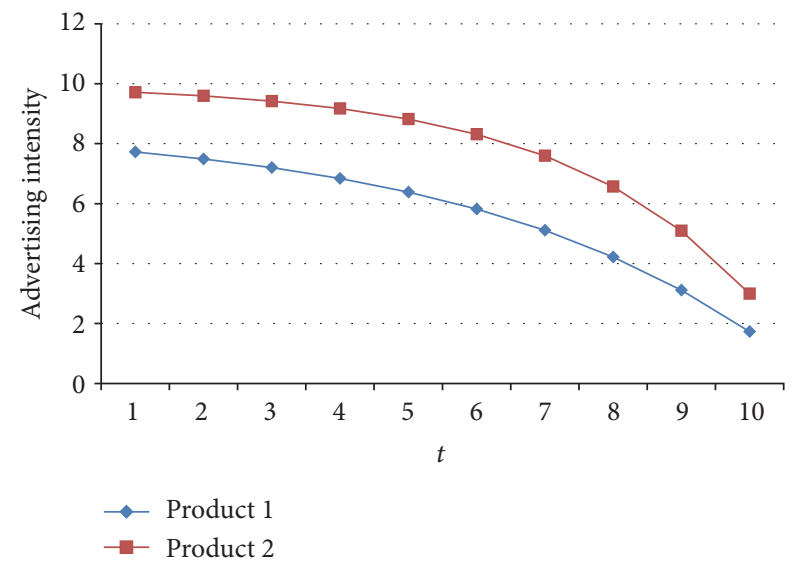

FIGURE 3: The advertising decisions under dynamic capacity strategy.

on advertisement and thus the demands increase from lower level. We found that the production capacities are all utilized for the dynamic strategy while some capacities are not used for the fixed strategy in the first three periods. In the middle periods from 4 to 9 , all capacities are sufficiently utilized under both dynamic and fixed strategies. However, as the dynamic strategy allocates more capacity in these periods and allows producing more quantities of products, more advertisement should be invested to promote more demands. That is why the advertising intensity under dynamic strategy (shown in Figure 3) is higher than that under fixed strategy (shown in Figure 4). In the last period, the capacity is also not sufficiently used for the fixed strategy. From the analysis, 


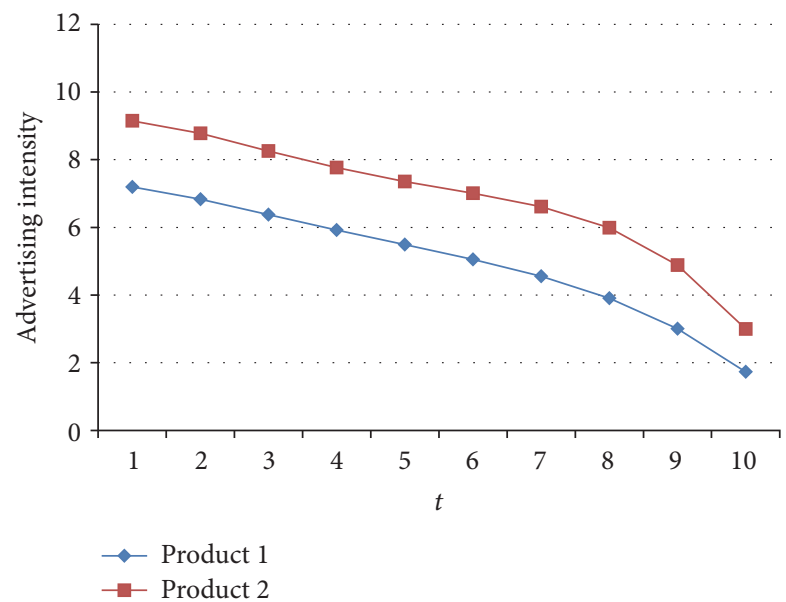

FIgURE 4: The advertising decisions under fixed capacity strategy.

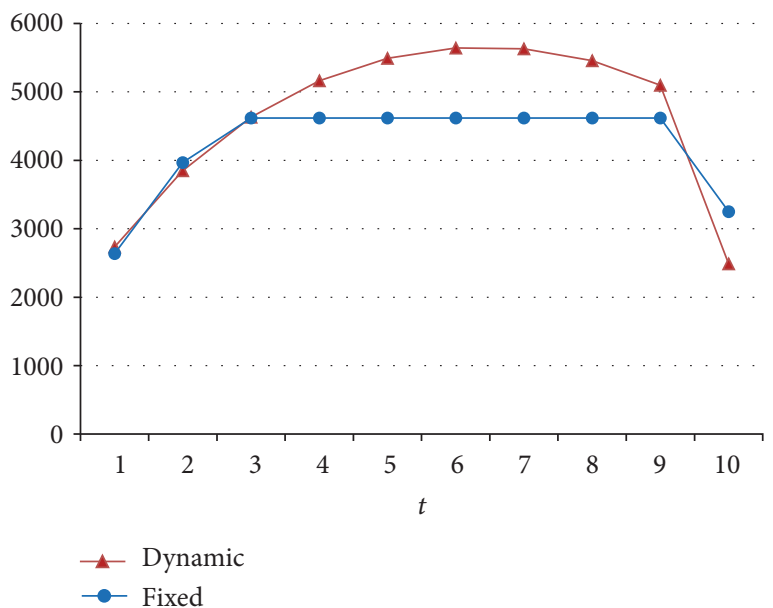

FIGURE 5: The actual consumption of production capacity under dynamic and fixed strategies.

we can see that some production capacities are not utilized under the fixed capacity strategy, and these capacities would not generate any profit.

\section{Conclusion}

Production/inventory management and dynamic advertisement are both important to the firms' operation. The presented study expands the literature on the integration of production planning and marketing efforts. Through the incorporation of classical newsvendor model with the N-A model, a nonlinear programming formulation is presented for the multiproduct multiperiod production planning problem with marketing effort. In the proposed model, the demand is assumed to be uncertain and depend on the accumulated investment on advertisement. Due to the computational complexity of the model, the Lagrangian relaxation and decomposition are employed to calculate the upper bound of the problem. We utilize the subgradient algorithm to search a tight upper bound of the model and a heuristic algorithm to find a feasible lower bound. Test results for hundreds of randomly generated instances show that the Lagrangian based solution approach can present very good solutions in short CPU time.

There are several future research directions for this paper. In this study, we considered the single budget constraint in each period, and one of the immediate extensions is to consider multiple capacity constraints in the production process. Also, we assume the demand linearly depends on the goodwill of customers on the product, while their relationship may be nonlinear in some situations. Thus, a fruitful avenue for future research is to consider other forms of demand functions, such as nonlinear forms, $D_{i t}=\alpha_{i t}(1-$ $\left.e^{-\beta_{i t} G_{i t}}\right)$, which is also widely used in literatures [30]. We use the N-A advertising model to capture the accumulating impact of advertisement, while there are some other advertising models in literature, such as Vidale-Wolfe model and the Bass diffusion model [24]. It would be also important to investigate the incorporation of production management model with other advertising models in future.

\section{Appendix}

Proof of Lemma 1. Function (17) can be further decomposed into $T$ subproblems as follows.

For $t=1,2, \ldots, T-1$,

$$
\begin{aligned}
\operatorname{NTP}_{i t}= & -s_{i t} \int_{A_{i t}}^{Z_{i t}}\left(Z_{i t}-u_{i t}\right) f_{i t}\left(u_{i t}\right) d u_{i t} \\
& -g_{i t} \int_{Z_{i t}}^{B_{i t}}\left(u_{i t}-Z_{i t}\right) f_{i t}\left(u_{i t}\right) d u_{i t} \\
& -Z_{i t}\left(\left(-\lambda_{t+1}+\lambda_{t}\right) h_{i}+\eta_{i, t+1}-\eta_{i t}\right),
\end{aligned}
$$

and for $t=T$,

$$
\begin{aligned}
& \mathrm{NTP}_{i T} \\
& =\int_{A_{i T}}^{Z_{i T}}\left[p_{i} u_{i T}-s_{i T}\left(Z_{i T}-u_{i T}\right)\right] f_{i T}\left(u_{i T}\right) d u_{i T} \\
& +\int_{Z_{i T}}^{B_{i T}}\left[p_{i} Z_{i T}-g_{i T}\left(u_{i T}-Z_{i T}\right)\right] f_{i T}\left(u_{i T}\right) d u_{i T} \\
& -\left(c_{i}+\lambda_{T} h_{i}-\eta_{i T}\right) Z_{i T}, \\
& \frac{d \mathrm{NTP}_{i t}}{d Z_{i t}} \\
& =-\left(s_{i t}+g_{i t}\right) F_{i t}\left(Z_{i t}\right)+g_{i t} \\
& -\left(\left(-\lambda_{t+1}+\lambda_{t}\right) h_{i}+\eta_{i, t+1}-\eta_{i t}\right), \\
& \frac{d^{2} \mathrm{NTP}_{i t}}{d Z_{i t}^{2}}=-\left(s_{i t}+g_{i t}\right) f_{i t}\left(Z_{i t}\right)<0 .
\end{aligned}
$$

Thus, $\mathrm{NTP}_{i t}$ is concave and its optimal solution can be obtained by solving $d \mathrm{NTP}_{i t} / d Z_{i t}=0$, which is

$$
Z_{i t}^{*}=\frac{g_{i t}+\left(\lambda_{t+1}-\lambda_{t}\right) h_{i}-\eta_{i, t+1}+\eta_{i t}}{g_{i t}+s_{i t}} .
$$


Similarly, $\mathrm{NTP}_{i T}$ is also concave and its optimal solution can be obtained by solving $d \mathrm{NTP}_{i T} / d Z_{i t}=0$, which is

$$
Z_{i T}^{*}=\frac{p_{i}+g_{i T}-c_{i}-\lambda_{T} h_{i}+\eta_{i T}}{p_{i}+g_{i T}+s_{i t}} .
$$

Proof of Proposition 2. We use backward recursive method to find the optimal solution for the dynamic advertising subproblem:

$$
C P=\min \left\{\sum_{t=1}^{T}\left(c u_{t}^{2}-\lambda_{t} \gamma A_{t}\right)(1+r)^{-(t-1)}\right\} .
$$

Let

$$
\begin{gathered}
a_{i t}=\alpha_{i t}\left(p_{i}-c_{i}-\lambda_{t} h_{i}+\eta_{i t}\right), \\
b_{i t}=\beta_{i t}\left(p_{i}-c_{i}-\lambda_{t} h_{i}+\eta_{i t}\right) .
\end{gathered}
$$

For period $T$,

$$
\mathrm{DAP}_{i, T}^{*}=\max \left\{a_{i T}+b_{i T} G_{i T}-w_{i} Y_{i T}^{2}\right\},
$$

and by the state function (7), we know $G_{i T}=Y_{i T}+(1-$ $\left.\delta_{i}\right) G_{i, T-1}$.

$$
\begin{aligned}
& \text { Then } \\
& \operatorname{DAP}_{i, T}^{*} \\
& =\max \left\{a_{i T}+b_{i T} Y_{i T}+b_{i T}\left(1-\delta_{i}\right) G_{i, T-1}-w_{i} Y_{i T}^{2}\right\} .
\end{aligned}
$$

From

$$
\frac{\partial \mathrm{DAP}_{i, T}}{\partial Y_{i T}}=b_{i T}-2 w_{i} Y_{i T}=0
$$

we obtain

$$
\begin{aligned}
Y_{i T}^{*} & =\frac{b_{i T}}{2 w_{i}}, \\
\mathrm{DAP}_{i, T}^{*} & =a_{i T}+\frac{b_{i T}^{2}}{4 w_{i}}+b_{i T}\left(1-\delta_{i}\right) G_{i, T-1} .
\end{aligned}
$$

For period $T-1$,

$$
\begin{aligned}
& \mathrm{DAP}_{i, T-1}^{*}=\max \left\{a_{i, T-1}+b_{i, T-1} G_{i, T-1}-w_{i} Y_{i, T-1}^{2}\right. \\
& \left.+L Z_{2, T}^{*}\right\}=\max \left\{a_{i, T-1}+b_{i, T-1} G_{i, T-1}-w_{i} Y_{i, T-1}^{2}\right. \\
& \left.+a_{i T}+\frac{b_{i T}^{2}}{4 w_{i}}+b_{i T}\left(1-\delta_{i}\right) G_{i, T-1}\right\}=\max \left\{a_{i, T-1}\right. \\
& +a_{i T}+\frac{b_{i T}^{2}}{4 w_{i}}+\left[b_{i, T-1}+b_{i T}\left(1-\delta_{i}\right)\right] G_{i, T-1} \\
& \left.+w_{i} Y_{i, T-1}^{2}\right\}=\max \left\{a_{i, T-1}+a_{i T}+\frac{b_{i T}^{2}}{4 w_{i}}\right. \\
& +\left[b_{i, T-1}+b_{i T}\left(1-\delta_{i}\right)\right]\left(Y_{i, T-1}+\left(1-\delta_{i}\right) G_{i, T-2}\right) \\
& \left.-w_{i} Y_{i, T-1}^{2}\right\} .
\end{aligned}
$$

From

$$
\frac{\partial \mathrm{DAP}_{i, T-1}^{*}}{\partial Y_{i, T-1}}=b_{i, T-1}+b_{i T}\left(1-\delta_{i}\right)-2 w_{i} Y_{i, T-1}=0,
$$

we obtain

$$
\begin{aligned}
Y_{i, T-1}^{*}= & \frac{b_{i, T-1}+b_{i T}\left(1-\delta_{i}\right)}{2 w_{i}} \\
\operatorname{DAP}_{i, T-1}^{*}= & a_{i, T-1}+a_{i T}+\frac{b_{i T}^{2}}{4 w_{i}} \\
& +\frac{\left(b_{i, T-1}+b_{i T}\left(1-\delta_{i}\right)\right)^{2}}{4 w_{i}} \\
& +\left[b_{i, T-1}+b_{i T}\left(1-\delta_{i}\right)\right]\left(1-\delta_{i}\right) G_{i, T-2} .
\end{aligned}
$$

Similarly,

for period $T-2, Y_{i, T-2}^{*}=\left(b_{i, T-2}+b_{i, T-1}\left(1-\delta_{i}\right)+b_{i T}(1-\right.$ $\left.\left.\delta_{i}\right)^{2}\right) / 2 w_{i}$

for period $T-3, Y_{i, T-3}^{*}=\left(b_{i, T-3}+b_{i, T-2}\left(1-\delta_{i}\right)+b_{i, T-1}(1-\right.$ $\left.\left.\delta_{i}\right)^{2}+b_{i T}\left(1-\delta_{i}\right)^{3}\right) / 2 w_{i}$;

for period $t, Y_{i t}^{*}=\left(1 / 2 w_{i}\right) \sum_{j=0}^{T-t} b_{i, t+j}\left(1-\delta_{i}\right)^{j}$.

Then we get all $Y_{i t}^{*}$ for $t=1,2, \ldots, T$.

Submit them into the state function, and we obtain the optimal goodwill $G_{i t}^{*}=\sum_{j=0}^{t-1}\left(1-\delta_{i}\right)^{j} Y_{i, t-j}^{*}+\left(1-\delta_{i}\right)^{t} G_{0}$.

\section{Notations}

Indices
$i$ : Index of products
$t$ : Index of periods.

\section{Parameters}

$p_{i}: \quad$ The selling price of product $i$

$c_{i}$ : The unit production cost of product $i$

$g_{i t}: \quad$ Backorder or penalty cost of one unit of product $i$ in period $t$

$s_{i t}$ : The holding cost of one unit of product $i$

in period $t$

$h_{i}$ : The rate of capacity usage for product $i$

$H_{t}$ : The total capacity available in period $t$

$u_{i t}$ : A stochastic variable with a known distribution defined on the range $\left[A_{i t}, B_{i t}\right]$

$f_{i t}\left(u_{i t}\right)$ : The p.d.f. of $u_{i t}$

$F_{i t}\left(u_{i t}\right)$ : The c.d.f. of $u_{i t}, F_{i t}\left(A_{i t}\right)=0$, and $F_{i t}\left(B_{i t}\right)=1$.

\section{Decision Variables}

$X_{i t}$ : The cumulative production quantity of product $i$ through period $t$

$Y_{i t}$ : The advertising intensity for product $i$ at period $t$

$Z_{i t}: Z_{i t}=X_{i t}-\sum_{j=1}^{t} D_{i j}$. 


\section{Competing Interests}

The authors declare that they have no competing interests.

\section{Acknowledgments}

This research is supported by National Natural Science Foundation of China (Grant no. 71201169).

\section{References}

[1] M. Khouja, "The single-period (news-vendor) problem: literature review and suggestions for future research," Omega, vol. 27, no. 5, pp. 537-553, 1999.

[2] N. C. Petruzzi and M. Dada, "Pricing and the newsvendor problem: a review with extensions," Operations Research, vol. 47, no. 2, pp. 183-194, 1999.

[3] Y. Qin, R. Wang, A. J. Vakharia, Y. Chen, and M. M. Seref, "The newsvendor problem: review and directions for future research," European Journal of Operational Research, vol. 213, no. 2, pp. 361-374, 2011.

[4] C. R. Sox and J. A. Muckstadt, "Multi-item, multi-period production planning with uncertain demand," IIE Transactions, vol. 28, no. 11, pp. 891-900, 1996.

[5] K. Matsuyama, "The multi-period newsboy problem," European Journal of Operational Research, vol. 171, no. 1, pp. 170-188, 2006.

[6] K. Kogan and V. Portougal, "Multi-period aggregate production planning in a news-vendor framework," Journal of the Operational Research Society, vol. 57, no. 4, pp. 423-433, 2006.

[7] A. Bensoussan, M. Çakanyildirim, and S. P. Sethi, "A multiperiod newsvendor problem with partially observed demand," Mathematics of Operations Research, vol. 32, no. 2, pp. 322-344, 2007.

[8] K. Pan, K. K. Lai, L. Liang, and S. C. H. Leung, "Two-period pricing and ordering policy for the dominant retailer in a twoechelon supply chain with demand uncertainty," Omega, vol. 37, no. 4, pp. 919-929, 2009.

[9] A. Bisi, M. Dada, and S. Tokdar, "A censored-data multiperiod inventory problem with newsvendor demand distributions," Manufacturing and Service Operations Management, vol. 13, no. 4, pp. 525-533, 2011.

[10] N. Xu, "Optimality of myopic inventory policy for a singleproduct, multi-period, stochastic inventory problem with batch ordering and capacity commitment," IIE Transactions, vol. 45, no. 8, pp. 925-938, 2013.

[11] Y. Zhang, V. Vovk, and W. G. Zhang, "Probability-free solutions to the non-stationary newsvendor problem," Annals of Operations Research, vol. 223, pp. 433-449, 2014.

[12] G. Kim, K. Wu, and E. Huang, "Optimal inventory control in a multi-period newsvendor problem with non-stationary demand," Advanced Engineering Informatics, vol. 29, no. 1, pp. 139-145, 2015.

[13] Y. Zhang and X. Yang, "Online ordering policies for a twoproduct, multi-period stationary newsvendor problem," Computers \& Operations Research, vol. 74, pp. 143-151, 2016.

[14] L. E. Cárdenas-Barrón, J. L. González-Velarde, and G. TreviñoGarza, "A new approach to solve the multi-product multiperiod inventory lot sizing with supplier selection problem," Computers \& Operations Research, vol. 64, pp. 225-232, 2015.
[15] F. M. Bass and D. G. Clarke, "Testing distributed lag models of advertising effect," Journal of Marketing Research, vol. 9, no. 3, pp. 298-308, 1972.

[16] P. S. H. Leeflang, G. M. Mijatovic, and J. Saunders, "Identification and estimation of complex multivariate lag structures: a nesting approach," Applied Economics, vol. 24, no. 2, pp. 273283, 1992.

[17] E. Kappe, A. S. Blank, and W. S. DeSarbo, "A general multiple distributed lag framework for estimating the dynamic effects of promotions," Management Science, vol. 60, no. 6, pp. 1489-1510, 2014.

[18] D. Berkowitz, A. Allaway, and G. D'Souza, "Estimating differential lag effects for multiple media across multiple stores," Journal of Advertising, vol. 30, no. 4, pp. 59-65, 2001.

[19] J. D. Herrington and W. A. Dempsey, "Comparing the current effects and carryover of national-, regional-, and local-sponsor advertising," Journal of Advertising Research, vol. 45, no. 1, pp. 60-72, 2005.

[20] R. Breuer, M. Brettel, and A. Engelen, "Incorporating longterm effects in determining the effectiveness of different types of online advertising," Marketing Letters, vol. 22, no. 4, pp. 327340, 2011.

[21] A. Aravindakshan and P. A. Naik, "How does awareness evolve when advertising stops? The role of memory," Marketing Letters, vol. 22, no. 3, pp. 315-326, 2011.

[22] N. Terui, M. Ban, and G. M. Allenby, "The effect of media advertising on brand consideration and choice," Marketing Science, vol. 30, no. 1, pp. 74-91, 2011.

[23] M. Nerlove and K. J. Arrow, "Optimal advertising policy under dynamic conditions," Economica, vol. 29, no. 114, pp. 129-142, 1962.

[24] J. Huang, M. Leng, and L. Liang, "Recent developments in dynamic advertising research," European Journal of Operational Research, vol. 220, no. 3, pp. 591-609, 2012.

[25] V. Srinivasan, "Decomposition of a multi-period media scheduling model in terms of single period equivalents," Management Science, vol. 23, no. 4, pp. 349-360, 1976.

[26] F. M. Bass, N. Bruce, S. Majumdar, and B. P. S. Murthi, "Wearout effects of different advertising themes: a dynamic Bayesian model of the advertising-sales relationship," Marketing Science, vol. 26, no. 2, pp. 179-195, 2007.

[27] G. M. Erickson, "A differential game model of the marketingoperations interface," European Journal of Operational Research, vol. 211, no. 2, pp. 394-402, 2011.

[28] A. Aravindakshan, O. Rubel, and O. Rutz, "Managing blood donations with marketing," Marketing Science, vol. 34, no. 2, pp. 269-280, 2015.

[29] S. Kraiselburd, V. G. Narayanan, and A. Raman, "Contracting in a supply chain with stochastic demand and substitute products," Production and Operations Management, vol. 13, no. 1, pp. 4662, 2004.

[30] C. Beltran-Royo, L. F. Escudero, and H. Zhang, "Multiperiod multiproduct advertising budgeting: stochastic optimization modeling," Omega, vol. 59, pp. 26-39, 2016. 


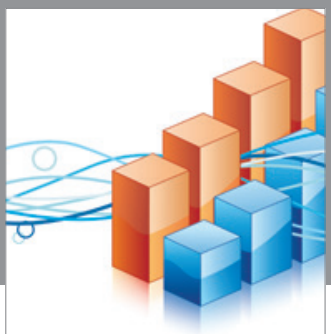

Advances in

Operations Research

vatem alat4

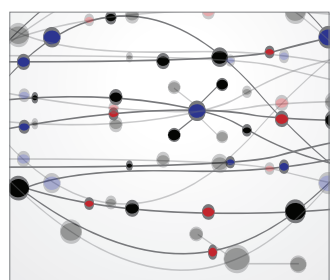

\section{The Scientific} World Journal
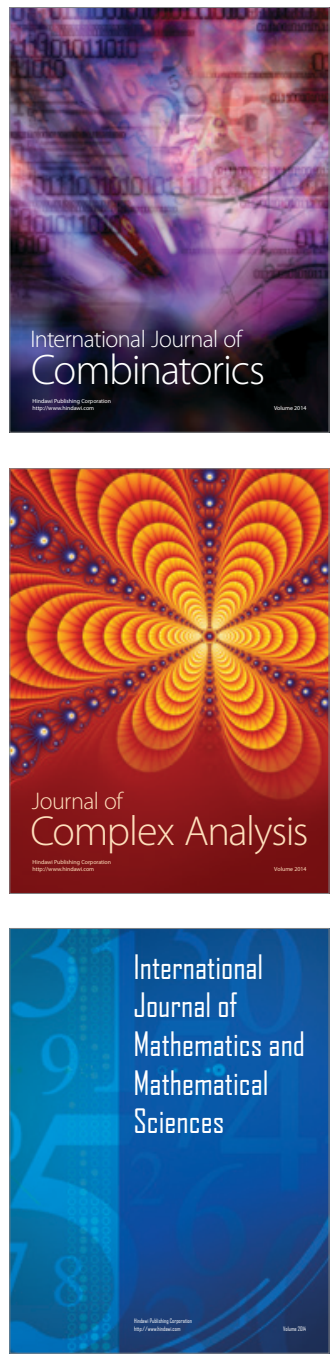
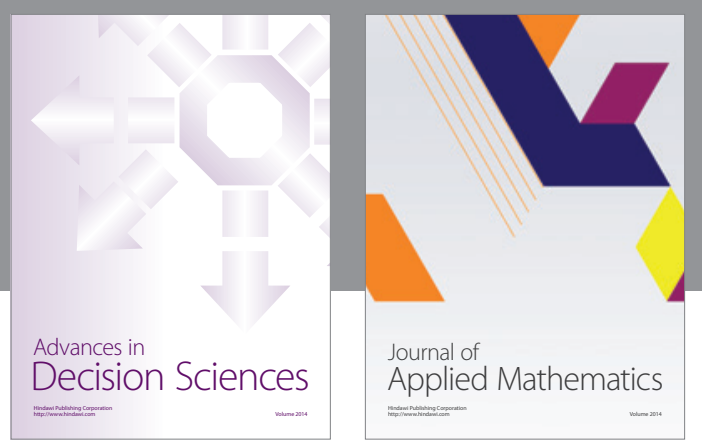

Algebra

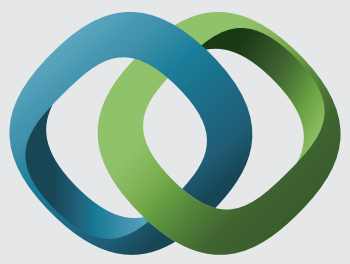

\section{Hindawi}

Submit your manuscripts at

http://www.hindawi.com
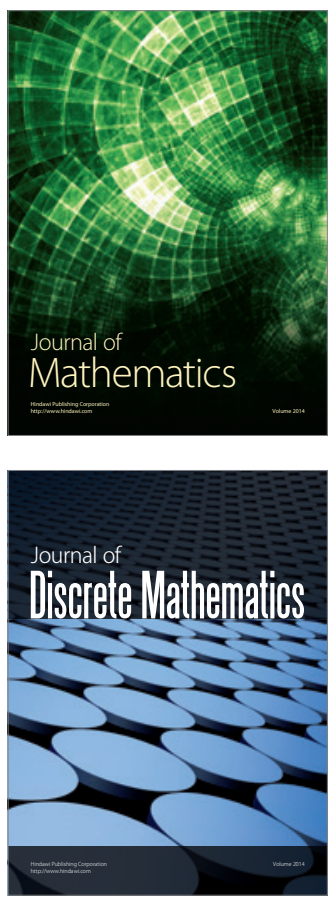

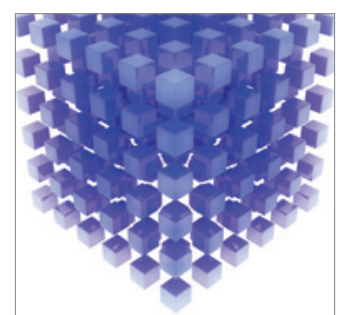

Mathematical Problems in Engineering
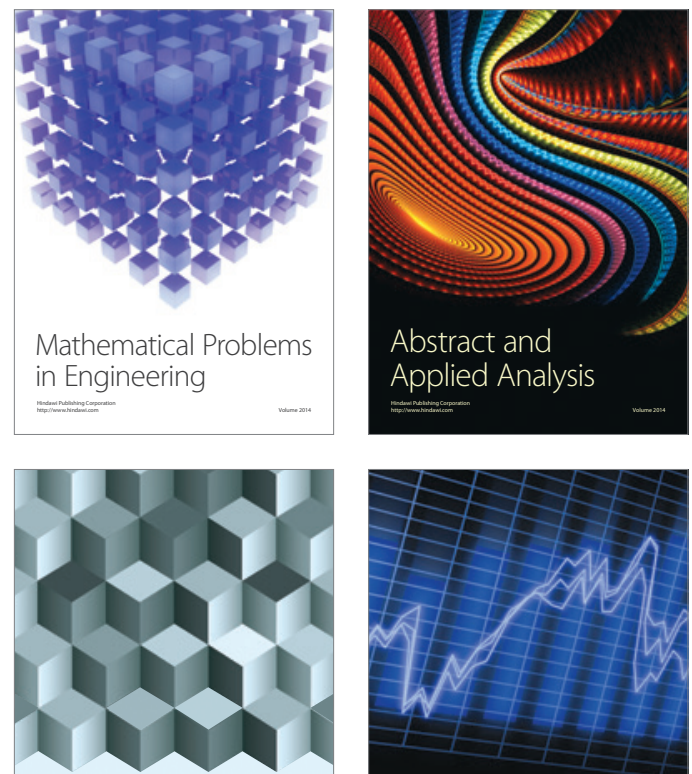

Journal of

Function Spaces

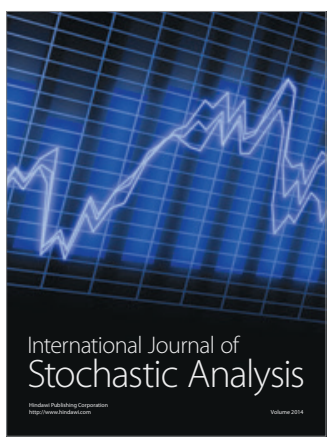

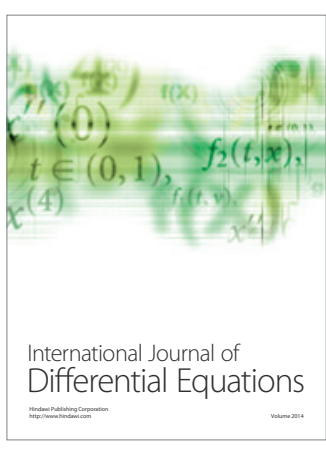
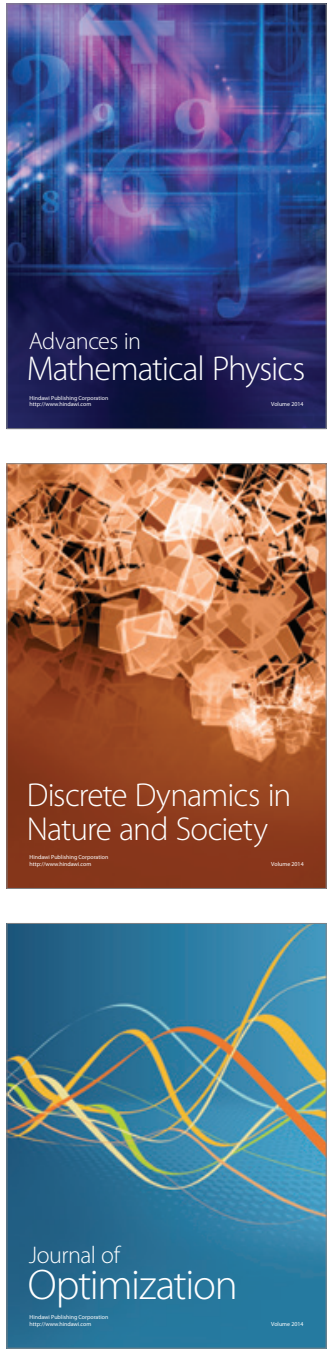